Chinese Journal of Organic Chemistry

ARTICLE

\title{
酸度调控的 3,3-二乙硫基丙烯酸酯的吲哚化反应
}

\author{
于海丰 $*, a$ 李铁纯 ${ }^{a}$ 廖沛球 ${ }^{b}$ 丁全平 ${ }^{a}$ 辛 广 $a$ 侯冬岩*, $a$ \\ $\left({ }^{a}\right.$ 鞍山师范学院化学与生命科学学院 鞍山 114007) \\ $\left({ }^{b}\right.$ 东北师范大学化学学院 长春 130024)
}

\begin{abstract}
摘要 探讨了酸度调控的 3,3-二乙硫基丙烯酸酯的吲哚化反应, 选择性地合成 3,3-二吲哚基丙烯酸酯和 3-吲哚基-3-氧 代丙酸酯. 研究表明, 3,3-二乙硫基丙烯酸酯与吲哚反应时, 在稀酸条件下, 高产率生成 3,3-二吲哚基丙烯酸酯, 而在浓 酸条件下, 生成的 3-吲哚基-3-乙硫基丙烯酸酯不稳定, 在后处理和柱层析分离时易水解, 高产率得到 3-吲哚基- 3-氧代 丙酸酯.
\end{abstract}

关键词 $\alpha$-羰基二硫缩烯酮; 3,3-二乙硫基丙烯酸酯; 吲哚衍生物; 亲核取代反应

\section{Acidity-Controlled Indolylation of 3,3-Bis(ethylthio)acrylate}

\author{
Yu, Haifeng*,a $\quad$ Li, Tiechun $^{a} \quad$ Liao, Peiqiu $^{b} \quad$ Diao, $^{*}$ Quanping $^{a}$ \\ Xin, Guang ${ }^{a} \quad$ Hou, Dongyan*,a \\ ( ${ }^{a}$ School of Chemistry and Life Science, Anshan Normal University, Anshan 114007) \\ ( ${ }^{b}$ Department of Chemistry, Northeast Normal University, Changchun 130024)
}

\begin{abstract}
The acidity controlled indolylation reaction of 3,3-bis(ethylthio)acrylates to selectively yield 3,3-di(indol-3yl)acrylates and 3-(indol-3-yl)-3-oxopropanoates was studied. It showed that 3,3-di(indol-3-yl)acrylates were obtained in good yield under diluted acidity condition when 3,3-bis(ethylthio)acrylates reacted with indole derivatives, while the reaction performed under concentrated acidity produced 3-ethylthio-3-(indol-3-yl)acrylate, which were easily hydrolyzed to supply 3-(indol-3-yl)-3-oxopropanoates in good yield under acid condition.
\end{abstract}

Keywords $\alpha$-oxo ketene dithioacetals; 3,3-bis(ethylthio)acrylates; indole derivatives; necleophilic substitution

吲哚衍生物是重要的一类有机化合物, 它们中有些 不仅具有良好的生物活性, 被用作治疗疾病的临床药 物 ${ }^{[1]}$, 而且有些结构简单的衍生物也是合成复杂吲哚衍 生物的重要合成中间体 ${ }^{[2,3]}$. 有机化合物的吲哚化反应 是合成吲哚衍生物的重要方法, 例如过渡金属催化的卤 代烃吲哚化能合成 2-或 3-烃基吲哚 ${ }^{[4,5]}$, 酸催化的联 烯 ${ }^{[6]}$ 、炔醇 ${ }^{[7]}$ 和 $\alpha, \beta$-不饱和羰基化合物 ${ }^{[8 \sim 10]}$ 等吲哚化反应 能合成 3-烷基吗哚衍生物, 经过 $\mathrm{C}-\mathrm{H}$ 活化偶联方式的 烯烃吲哚化可有效合成 2-或 3-烯基化吲哚 ${ }^{[11,12]}$, 酸䣶或 酰氯的吅哚化反应能合成 3-酰基吲哚衍生物等 ${ }^{[13]}$. 为探 索简洁有效的合成方法和新颖的吲哚衍生物, 有机化合 物的吲哚化反应一直受到合成化学家的广泛关注.

易制备和多反应中心的 $\alpha$-羰基二硫缩烯酮是重要

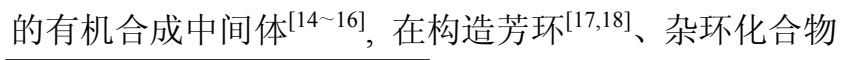

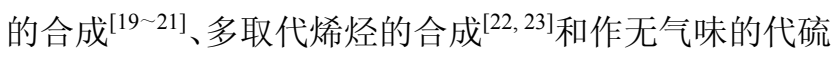
醇试剂 ${ }^{[24 ~ 28]}$ 中有广泛的应用. 最近, 在拓展 $\alpha$-羰基二硫 缩烯酮在合成的应用中, 我们进行了三氟乙酸(TFA)作 用的 $\alpha$-羰基二硫缩烯酮的吲哚化反应研究, 已系统研究 3,3-二乙硫基丙烯酮(1a)的吲哚化反应(Scheme 1A), 重 点考察了反应体系的酸度强弱和吲哚的 2 位取代基对反 应的影响 ${ }^{[29,30]}$. 当 $1 \mathbf{a}$ 与 2 位非取代的吲哚反应时, 在浓 酸性条件下生成稳定的 $\beta$-弜哚基- $\alpha, \beta$-不饱和酮, 而在稀 酸性条件下主要得到 $\beta, \beta$-二吲哚基- $\alpha, \beta$-不饱和酮衍生物 ${ }^{[29]}$; 当 $1 \mathbf{a}$ 与 2 位取代的吲哚反应时, 如果取代基是甲 基, 通过控制吲哚的量和反应时间, 能选择性得到稳定 的 $\beta$-吲哚基- $\alpha, \beta$-不饱和酮和 $\beta, \beta$-二吲哚基- $\alpha, \beta$-不饱和酮 衍生物, 而取代基为苯基时, 无论增加吲哚的量还是延 长反应时间, 都只生成稳定的 $\beta$-吲哚基- $\alpha, \beta$-不饱和

*E-mail: yuhf68105@sina.com

Received December 16, 2013; revised January 6, 2014; published online January 16, 2014.

Project supported by the National Natural Science Foundations of China (No. 20272008) and the Science Foundation of Anshan City (Nos. 2011MS44, 2012KJ02).

国家自然科学基金(No. 20272008)和鞍山市科技基金(Nos. 2011MS44, 2012KJ02)资助项目. 
酮 ${ }^{[30]}$. 在研究中, 我们也初步研究了 3,3-二乙硫基丙烯 酸酯(1b)的吲哚化反应. 与 $1 \mathrm{a}$ 的吲哚化反应不同, 在浓 酸条件下 $1 \mathbf{b}$ 的吲哚化反应生成的 3-吲哚基-3-乙硫基丙 烯酸酯不稳定, 在后处理和柱层析分离中易水解, 生成 稳定的 3-吲哚基-3-氧代丙酸酯. 作为系列工作, 在以前 工作基础上, 本文详细研究 $\mathbf{1 b}$ 的吲哚化反应(Scheme 1B).

(A) 3,3-二乙硫基丙烯酮的吲哚化反应(以前工作)

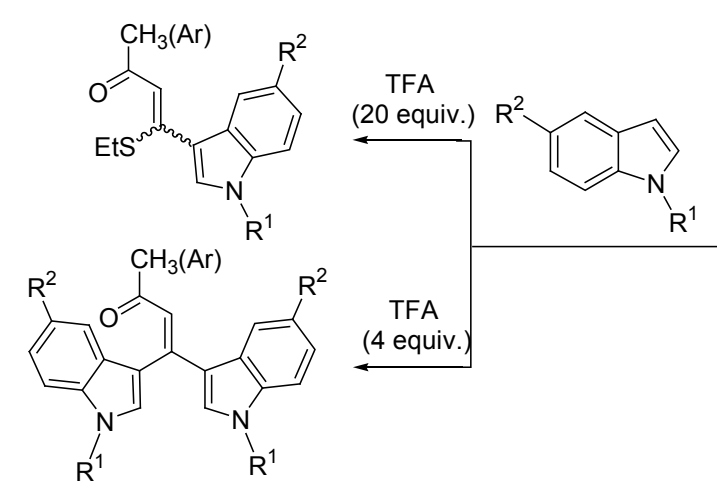

\section{1 结果与讨论}

\section{1 反应条件优化}

根据以前的工作, 用 TFA- $\mathrm{CH}_{2} \mathrm{Cl}_{2}$ 体系研究 3,3-二 乙硫基丙烯酸酯(1b)的吲哚化反应. 以 $1 \mathrm{ba}$ 和 $2 \mathrm{a}$ 的反应 为例, 对反应条件进行优化, 结果如表 1 所示.

(B) 3,3-二乙硫基丙烯酸酯的吲哚化反应(本文工作)

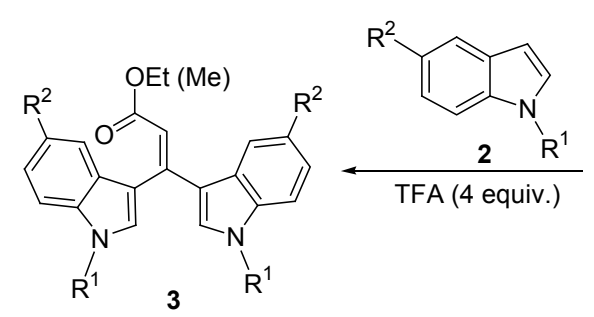

$$
\text { . }
$$

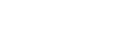

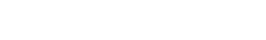

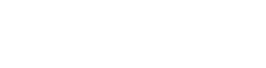

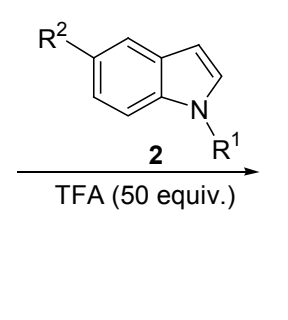
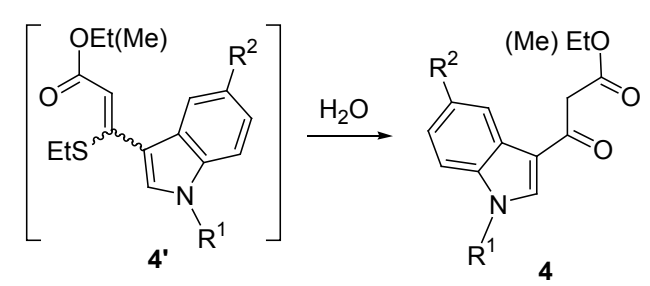

Scheme 1

表 1 反应条件优化 ${ }^{a}$

Table 1 Screening of reaction conditions<smiles>CCOC(=O)C=C(c1c[nH]c2ccccc12)c1c[nH]c2ccccc12</smiles>

condition<smiles>CCOC(=O)C=C(CC)CC</smiles><smiles>Cc1cc2ccccc2[nH]1</smiles><smiles>CCOC(=O)C=C(CC)c1c[nH]c2ccccc12</smiles><smiles>CCOC(=O)CC(=O)c1c[nH]c2ccccc12</smiles>

4'a

\begin{tabular}{|c|c|c|c|c|c|}
\hline \multirow{2}{*}{ Entry } & \multirow{2}{*}{ 1ba : 2a : TFA (molar ratio) } & \multirow{2}{*}{ Temp. $/{ }^{\circ} \mathrm{C}$} & \multirow{2}{*}{ Time/h } & \multicolumn{2}{|c|}{ Yield/\% } \\
\hline & & & & $3 \mathbf{a}$ & $4 \mathbf{a}$ \\
\hline 1 & $1: 2: 4$ & Reflux & 2.0 & 90 & - \\
\hline 2 & $1: 1: 20$ & Reflux & 2.5 & 23 & 41 \\
\hline 3 & $1: 1: 20$ & r.t & 10 & 20 & 47 \\
\hline 4 & $1: 1: 30$ & r.t & 7.5 & 14 & 63 \\
\hline 5 & $1: 1: 40$ & r.t & 3.5 & 10 & 75 \\
\hline 6 & $1: 1: 50$ & r.t & 2.0 & - & 85 \\
\hline 7 & $1: 1: 60$ & r.t & 1.5 & - & 86 \\
\hline 8 & $1: 1: 50$ & Reflux & 0.5 & 7 & 77 \\
\hline
\end{tabular}

反应条件: 1 ba $(0.25 \mathrm{mmol}), \mathrm{CH}_{2} \mathrm{Cl}_{2}(2 \mathrm{~mL})$. 
根据前期工作的最佳反应条件 ${ }^{[29]}$, 在回流条件下, $1 \mathbf{b a}, 2 \mathbf{a}$ 和 TFA 的物质的量比为 $1: 2: 4$ 时, $1 \mathbf{b a}$ 和 $2 \mathbf{a}$ 能有效进行反应, 高产率制得 3,3-二吲哚基丙烯酸乙酯 3a (表 1, Entry 1). 然而, 当 1ba, 2a 和 TFA 的物质的量 比为 $1: 1: 20$ 时, 回流条件下, 在生成 $41 \% \mathbf{4 a}$ 的同时, 也得到 23\% 3a, 即使在常温条件下反应，仍有 $20 \% 3 \mathbf{a}$ 生成(表 1, Entries 2, 3), 所以在研究中重点优化合成 4a 的最佳反应条件. 研究表明, 随着反应体系酸性的增 强, 产物 $\mathbf{4 a}$ 的产率增加, 3a 的产率减少(表 1, Entries 4, 5). 当 $1 \mathrm{ba}, 2 \mathrm{a}$ 和 TFA 的物质的量比为 $1: 1: 50$ 时, $4 \mathrm{a}$ 的产率高达 $85 \%$, 只有痕量的 3a 生成(表 1, Entry 6). 继续增加酸的量, 反应结果变化不大(表 1, Entry 7). 保持 1ba, 2a 和 TFA 的物质的量比为 $1: 1: 50$, 回流条 件下进行该反应时，生成 77\% 4a 和 7\% 3a (表 1, Entry 8). 因此, 在 1ba, 2a 和 TFA 的物质的量比为 $1: 2: 4$ 时, 在回流条件下, 合成 3,3-二吲哚基丙烯酸酯(3); 在 1ba, 2a 和 TFA 的物质的量比为 $1: 1: 50$ 时, 常温条件 下，制备 3-吲哚基-3-氧代丙酸酯(4).

\section{$1.23,3$-二乙硫基丙烯酸酯的吲哚化反应}

在最佳反应条件下, $\mathbf{1 b}$ 与 $\mathbf{2}$ 有效进行亲核取代反应. 当 $1 \mathrm{~b}$ 、吲哚 2 和 TFA 的物质的量比为 $1: 2: 4$ 时, 回 流条件下, 可高产率生成 3,3-二吲哚基丙烯酸酯衍生物 $3 \mathbf{a} \sim 3 \mathrm{~h}$ (表 2); 当 $\mathbf{1 b}$ 、吲哚 2 和 TFA 的物质的量比为 1 : $1: 50$ 时, 虽然反应能生成3-吲哚基-3-烷硫基丙烯酸酯 $4^{\prime}$, 但 $4^{\prime}$ 常温不稳定, 在后处理和柱层析提纯时, 易水 解生成稳定的 3-吲哚基-3-氧代丙酸酯 $4 \mathbf{a} \sim 4 \mathrm{~h}$ (表 2).

\section{3 反应机理研究}

根据前期工作 ${ }^{[29,30]}$ 和上述实验结果，提出如下反应 机理(Scheme 2). 在酸作用下, $\mathbf{1 b a}$ 质子化生成中间体 I 后, 与吲哚 2a 反应, 得到中间体 II. 研究表明, 在酸性 溶液中, 存在吲哚 $\mathbf{2 a}$ 、质子化吲哚 $\mathbf{A}$ 和二聚吲哚 $\mathbf{B}$ 之 间的平衡 (Scheme 3) ${ }^{[31]}$.

在稀酸性溶液中, 吲哚 2a 主要以二聚吲哚 $\mathbf{B}$ 的形 式存在, 它受热易分解成吲哚 2a. 分解生成的 2a 进攻 中间体 II 的互变异构体 III, 脱去乙硫醇后, 得到 3,3二吲哚基丙烯酸乙酯(3a). 在浓酸性溶液中, 吲哚主要 以质子化吲哚 $\mathbf{A}$ 形式存在, 它的亲核性比 $\mathbf{2 a}$ 低, 难进一 步进攻中间体 III, 生成 3a, 而是 III 的异构体 II 脱去质 子, 生成 3-吲哚基-3-烷硫基丙烯酸乙酯 4'a. 在酸性条 件下, $\mathbf{4} \mathbf{a}$ 不稳定, 易水解生成 3-吲哚基-3-氧代丙酸乙酯 (4a).

\section{2 结论}

在三氟乙酸(TFA)存在下, 通过调控反应体系的酸 度, 3,3-二乙硫基丙烯酸酯的吲哚化可选择性制备 3,3-二

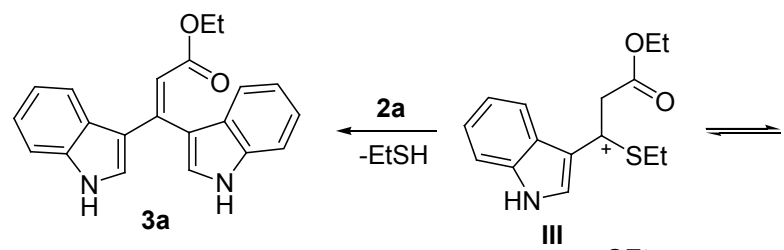

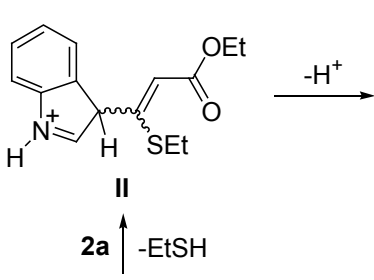<smiles>CCOC(=O)C=C(CC)c1c[nH]c2ccccc12</smiles>
4'a

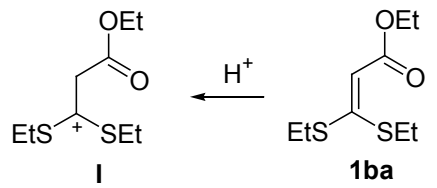
-EtSH $\mathrm{H}_{2} \mathrm{O}, \mathrm{H}^{+}$<smiles>CCOC(=O)CC(=O)c1c[nH]c2ccccc12</smiles>

Scheme 2
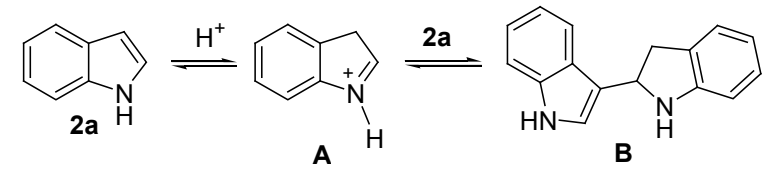

Scheme 3

吲哚基丙烯酸酯(3)和 3-吲哚基-3-乙硫基丙烯酸酯(4'). 然而, 4 不稳定，在后处理和柱层析分离时易水解，最终 高产率得到 3-吲哚基-3-氧代丙酸酯(4).

\section{3 实验部分}

\section{1 仪器与试剂}

XRC-1 显微熔点测定仪 (温度计未经校正); Unity-600 或 400 核磁共振仪 ( $\mathrm{CDCl}_{3}$ 溶剂, TMS 为内标); PE-2400 自动元素分析仪. 所用试剂均为分析纯.

\section{2 实验方法}

\subsubsection{3,3-二吲哚基丙烯酸酯的合成}

向 $25 \mathrm{~mL}$ 圆底烧瓶中加入 $\mathbf{1 b a}(55 \mathrm{mg}, 0.25 \mathrm{mmol})$, 吲哚 $2 \mathrm{a}(59 \mathrm{mg}, 0.50 \mathrm{mmol}), 2 \mathrm{~mL}$ 二氯甲烷和三氟乙酸 $(0.074 \mathrm{~mL}, 1 \mathrm{mmol})$. 回流 $2 \mathrm{~h}, \mathrm{TLC}$ 检测底物消失后，向 反应液中加入 $20 \mathrm{~mL}$ 水, 用二氯甲烷萃取 $(5 \mathrm{~mL} \times 3)$, 有 机相用 $10 \mathrm{~mL} \mathrm{NaHCO}$ 饱和溶液洗涤, $\mathrm{MgSO}_{4}$ 干燥, 柱 层析[洗脱液: $V$ (石油醚) $： V($ 乙酸乙酯 $)=4: 1$ ] 后, 得到 白色晶体 3a (74 mg)，产率 90\%. 用相同方法合成 3b $3 \mathbf{h}$, 结果列于表 2 中.

\section{2 .23 -吲哚基-3-氧代丙酸酯的合成}

向 $25 \mathrm{~mL}$ 圆底烧瓶中加入 $1 \mathrm{ba}(55 \mathrm{mg}, 0.25 \mathrm{mmol})$, 吲哚 $2 \mathbf{a}(30 \mathrm{mg}, 0.25 \mathrm{mmol}), 2 \mathrm{~mL}$ 二氯甲烷和三氟乙酸 (0.93 mL, $12.5 \mathrm{mmol})$. 常温摚拌 $2 \mathrm{~h}, \mathrm{TLC}$ 检测底物消失 后，向反应液中加入 $20 \mathrm{~mL}$ 水，用二氯甲烷萃取 (5 
表 23 ,3-二乙硫基丙烯酸酯 $\mathbf{1 b}$ 的吲哚化反应 ${ }^{a}$

Table 2 Indolylation of 3,3-bis(ethylthio)acrylates $\mathbf{1 b}$
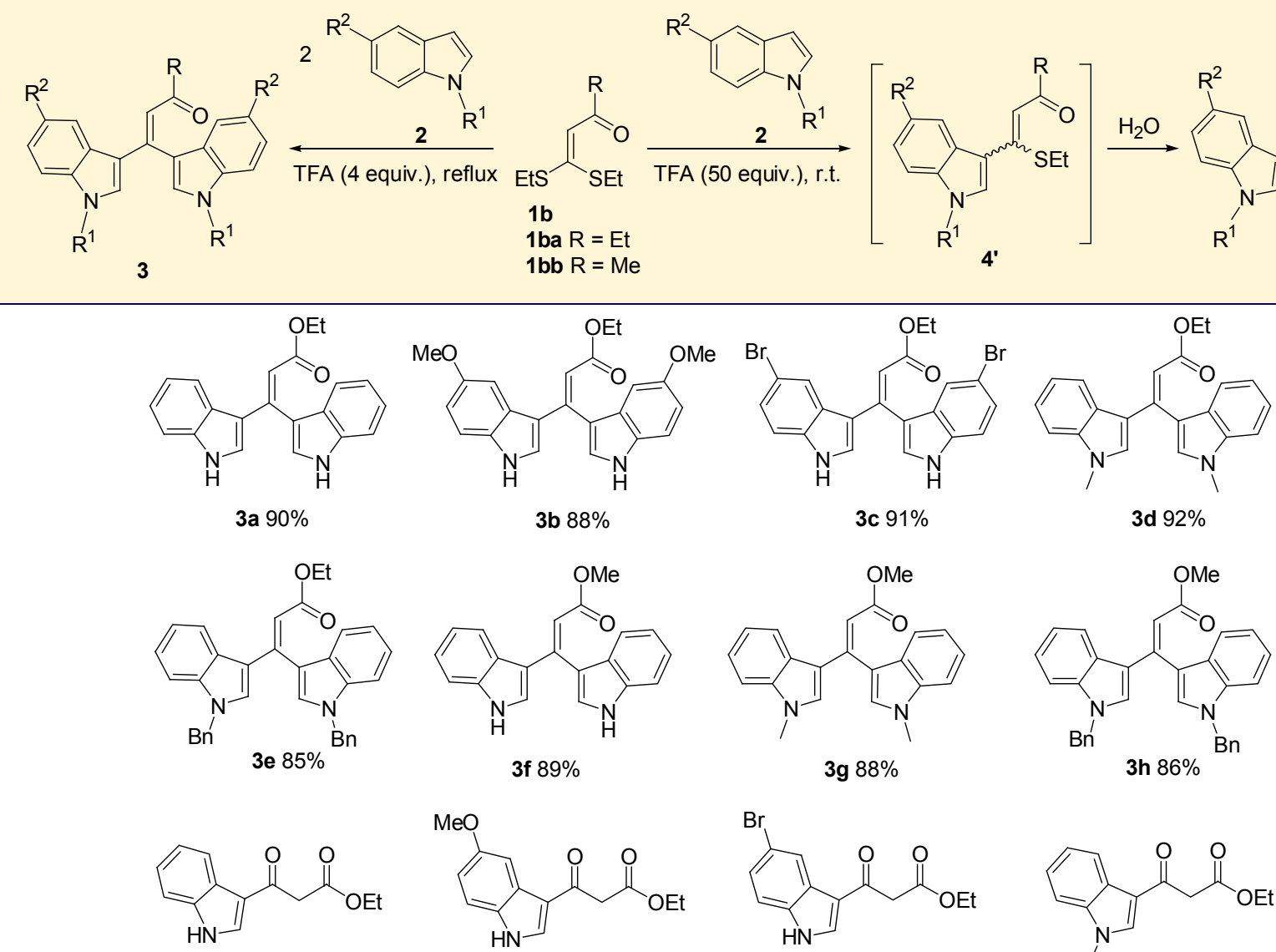

4a $85 \%$

4b $85 \%$<smiles>CCOC(=O)CC(=O)c1c[nH]c2ccc(Br)cc12</smiles>

4c $87 \%$<smiles>CCOC(=O)CC(=O)c1cn(C)c2ccccc12</smiles><smiles>CCOC(=O)CC(=O)c1cn(Cc2ccccc2)c2ccccc12</smiles><smiles>COC(=O)CC(=O)c1c[nH]c2ccccc12</smiles>

4f $85 \%$

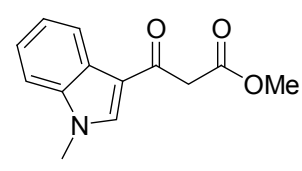

$4 g 88 \%$

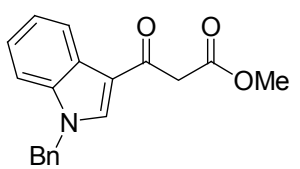

4h $87 \%$

${ }^{a}$ Isolated yield.

$\mathrm{mL} \times 3)$. 加入 $1 \mathrm{~g}$ 硅胶后, 室温放置 $12 \mathrm{~h}$ 至溶剂消失后, 柱层析 [洗脱液: $V$ (石油醚) $: V$ (乙酸乙 酯 $)=6: 1$ ], 得 到白色晶体 4a (49 mg), 产率 $85 \%$. 用相同方法合成 $4 b \sim 4 h$, 结果列于表 2 中.

\section{3 化合物表征}

化合物 1b, 3a, 3d, 4a, 4d 是已知化合物, ${ }^{1} \mathrm{H}$ NMR 和 ${ }^{13} \mathrm{C}$ NMR 的数据与文献[29]报道一致.

3,3-二(5-甲氧基吲哚基)丙烯酸乙酯(3b): 白色晶 体, m.p. 84 86 ${ }^{\circ} \mathrm{C} ;{ }^{1} \mathrm{H}$ NMR (600 MHz, $\left.\mathrm{CDCl}_{3}\right) \delta: 1.14$ $(\mathrm{t}, J=7.1 \mathrm{~Hz}, 3 \mathrm{H}), 3.65(\mathrm{~s}, 3 \mathrm{H}), 3.77(\mathrm{~s}, 3 \mathrm{H}), 4.07 \sim 4.11$ (q, $J=7.1 \mathrm{~Hz}, 2 \mathrm{H}), 6.43(\mathrm{~s}, 1 \mathrm{H}), 6.72(\mathrm{~d}, J=8.8 \mathrm{~Hz}, 1 \mathrm{H})$, $6.76(\mathrm{~s}, 1 \mathrm{H}), 6.81(\mathrm{~s}, 1 \mathrm{H}), 6.85(\mathrm{~d}, J=8.8 \mathrm{~Hz}, 1 \mathrm{H}), 6.89$ (s, $1 \mathrm{H}), 7.02$ (d, $J=8.8 \mathrm{~Hz}, 1 \mathrm{H}), 7.15$ (s, $J=8.8 \mathrm{~Hz}, 1 \mathrm{H}), 7.21$ $(\mathrm{s}, 1 \mathrm{H}), 8.42(\mathrm{~s}, 1 \mathrm{H}), 8.55(\mathrm{~s}, 1 \mathrm{H}) ;{ }^{13} \mathrm{C}$ NMR $(150 \mathrm{MHz}$,
$\left.\mathrm{CDCl}_{3}\right) \delta: 14.3,55.8,56.0,59.8,102.3,103.3,110.6$, $112.2,112.2,112.3,112.4,115.1,118.7,126.2,127.5$, $127.6,129.7,131.1,132.2,145.3,154.2,155.0,168.1$. Anal. calcd for $\mathrm{C}_{23} \mathrm{H}_{22} \mathrm{~N}_{2} \mathrm{O}_{4}$ : C 70.75, $\mathrm{H}$ 5.68, N 7.17; found $\mathrm{C} 70.88, \mathrm{H}$ 5.71, N 7.25.

3,3-二(5-溴吲哚基)丙烯酸乙酯(3c): 白色晶体, m.p. 109 $111{ }^{\circ} \mathrm{C} ;{ }^{1} \mathrm{H}$ NMR $\left(600 \mathrm{MHz}, \mathrm{CDCl}_{3}\right) \delta: 1.23(\mathrm{t}, J=$ $7.1 \mathrm{~Hz}, 3 \mathrm{H}), 4.13 \sim 4.16(\mathrm{q}, J=7.1 \mathrm{~Hz}, 2 \mathrm{H}), 6.47(\mathrm{~s}, 1 \mathrm{H})$, $7.00(\mathrm{~s}, 1 \mathrm{H}), 7.11(\mathrm{~d}, J=8.5 \mathrm{~Hz}, 1 \mathrm{H}), 7.12(\mathrm{~s}, 1 \mathrm{H}), 7.18$ (d, $J=8.7 \mathrm{~Hz}, 1 \mathrm{H}), 7.25(\mathrm{~d}, J=8.7 \mathrm{~Hz}, 1 \mathrm{H}), 7.34$ (d, $J=8.5$ Hz, 1H), 7.39 (s, 1H), 7.94 (s, 1H), 8.45 (s, 1H), 8.59 (s $1 \mathrm{H}) ;{ }^{13} \mathrm{C} \mathrm{NMR}\left(150 \mathrm{MHz}, \mathrm{CDCl}_{3}\right) \delta: 14.3,60.0,112.9$, $113.0,113.1,113.4,114.5,114.6,118.7,122.7,123.2$, $125.0,125.9,127.0,127.4,128.6,129.3,134.5,135.6$, 
143.0, 167.3. Anal. calcd for $\mathrm{C}_{21} \mathrm{H}_{16} \mathrm{Br}_{2} \mathrm{~N}_{2} \mathrm{O}_{2}$ : C 51.67, $\mathrm{H}$ 3.30, N 5.74; found C 51.78, H 3.24, N 5.66.

3,3-二(1-苠基吲哚基)丙烯酸乙酯(3e): 白色晶体, m.p. $127 \sim 129{ }^{\circ} \mathrm{C} ;{ }^{1} \mathrm{H}$ NMR $\left(600 \mathrm{MHz}, \mathrm{CDCl}_{3}\right) \delta: 1.02(\mathrm{t}$, $J=7.1 \mathrm{~Hz}, 3 \mathrm{H}), 4.01 \sim 4.04(\mathrm{q}, J=7.1 \mathrm{~Hz}, 2 \mathrm{H}), 5.21(\mathrm{~s}$, $1 \mathrm{H}), 5.33(\mathrm{~s}, 1 \mathrm{H}), 6.48(\mathrm{~s}, 1 \mathrm{H}), 7.04(\mathrm{t}, J=7.5 \mathrm{~Hz}, 1 \mathrm{H})$, $7.06(\mathrm{~d}, J=7.5 \mathrm{~Hz}, 1 \mathrm{H}), 7.12(\mathrm{~s}, 1 \mathrm{H}), 7.14 \sim 7.21(\mathrm{~m}, 6 \mathrm{H})$, $7.24 \sim 7.29$ (m, 8H), 7.32 (s, 1H), 7.36 (d, $J=8.0 \mathrm{~Hz}, 1 \mathrm{H})$, $7.77(\mathrm{~d}, J=8.0 \mathrm{~Hz}, 1 \mathrm{H}) ;{ }^{13} \mathrm{C} \mathrm{NMR}\left(150 \mathrm{MHz}, \mathrm{CDCl}_{3}\right) \delta$ : 13.8, 49.9, 50.0, 59.2, 109.6, 109.9, 111.9, 114.5, 118.3, $119.6,120.5,120.6,120.8,121.6,122.3,126.3,126.4$ (2C), 126.5 (2C), 127.3, 127.5, 127.9, 128.4 (2C), 128.5 (2C), 130.2, 131.6, 136.0, 136.3, 136.9, 137.1, 143.2, 167.0. Anal. calcd for $\mathrm{C}_{35} \mathrm{H}_{30} \mathrm{~N}_{2} \mathrm{O}_{2}$ : C 82.33, $\mathrm{H} 5.92, \mathrm{~N}$ 5.49; found C 82.18, H 5.99, N 5.40.

3,3-二吲哚基丙烯酸甲酯 (3f): 白色晶体, m.p. $182 \sim 184{ }^{\circ} \mathrm{C} ;{ }^{1} \mathrm{H}$ NMR $\left(600 \mathrm{MHz}, \mathrm{CDCl}_{3}\right) \delta: 3.63(\mathrm{~s}, 3 \mathrm{H})$, $6.55(\mathrm{~s}, 1 \mathrm{H}), 6.95 \sim 6.97(\mathrm{~m}, 2 \mathrm{H}), 7.00 \sim 7.03(\mathrm{~m}, 2 \mathrm{H})$, $7.12 \sim 7.20(\mathrm{~m}, 3 \mathrm{H}), 7.28 \sim 7.33(\mathrm{~m}, 3 \mathrm{H}), 8.31(\mathrm{~s}, 1 \mathrm{H})$, $8.33(\mathrm{~s}, 1 \mathrm{H}) ;{ }^{13} \mathrm{C} \mathrm{NMR}\left(150 \mathrm{MHz}, \mathrm{CDCl}_{3}\right) \delta: 51.0,111.1$, $111.3,111.6,119.1,119.7,120.0,120.6,120.8,121.1$, 121.8 (2C), 122.1, 122.9, 126.5, 135.9, 136.6, 137.0, 144.8, 167.7. Anal. calcd for $\mathrm{C}_{20} \mathrm{H}_{16} \mathrm{~N}_{2} \mathrm{O}_{2}$ : C 75.93, H 5.10, N 8.86; found C 76.01, H 5.03, N 8.94.

3,3-二(1-甲基吲哚基)丙烯酸甲酯(3g): 白色晶体, m.p. $131 \sim 133{ }^{\circ} \mathrm{C} ;{ }^{1} \mathrm{H}$ NMR $\left(600 \mathrm{MHz}, \mathrm{CDCl}_{3}\right) \delta$ : 3.59 $(\mathrm{s}, 3 \mathrm{H}), 3.66(\mathrm{~s}, 3 \mathrm{H}), 3.81(\mathrm{~s}, 3 \mathrm{H}), 6.47(\mathrm{~s}, 1 \mathrm{H}), 6.98(\mathrm{~s}$, $1 \mathrm{H}), 7.02(\mathrm{t}, J=7.3 \mathrm{~Hz}, 1 \mathrm{H}), 7.16 \sim 7.21(\mathrm{~m}, 2 \mathrm{H}), 7.25(\mathrm{~s}$, $1 \mathrm{H}), 7.28 \sim 7.33(\mathrm{~m}, 4 \mathrm{H}), 7.83(\mathrm{~d}, J=7.3 \mathrm{~Hz}, 1 \mathrm{H}) ;{ }^{13} \mathrm{C}$ NMR (150 MHz, $\left.\mathrm{CDCl}_{3}\right) \delta: 33.1,33.1,50.9,109.5,109.8$, $110.9,113.8,118.1,119.8,120.8,120.8,121.0,121.7$, $122.5,126.4,128.0,131.2$, 133.0, 136.8, 137.9, 144.6, 167.6. Anal. calcd for $\mathrm{C}_{22} \mathrm{H}_{20} \mathrm{~N}_{2} \mathrm{O}_{2}$ : C 76.72, $\mathrm{H}$ 5.85, N 8.13; found $\mathrm{C} 76.51, \mathrm{H} 5.75, \mathrm{~N} 8.05$.

3,3-二(1-苠基吲哚基)丙烯酸甲酯(3h)：白色晶体， m.p. $60 \sim 62{ }^{\circ} \mathrm{C} ;{ }^{1} \mathrm{H}$ NMR $\left(600 \mathrm{MHz}, \mathrm{CDCl}_{3}\right) \delta$ : 3.57 (s, $3 \mathrm{H}), 5.20(\mathrm{~s}, 2 \mathrm{H}), 5.33(\mathrm{~s}, 2 \mathrm{H}), 6.49(\mathrm{~s}, 1 \mathrm{H}), 7.02 \sim 7.36$ $(\mathrm{m}, 19 \mathrm{H}), 7.80(\mathrm{~s}, 1 \mathrm{H}) ;{ }^{13} \mathrm{C}$ NMR $\left(150 \mathrm{MHz}, \mathrm{CDCl}_{3}\right) \delta$ : 50.3, 50.4, 50.9, 110.0, 110.3, 111.6, 114.4, 118.5, 120.0, $120.9,121.0,121.1,121.9,122.7,126.7,126.8$ (2C), 126.9 (2C), 127.7, 127.9, 128.2, 128.8 (2C), 128.9 (2C), 130.8, 132.2, 136.4, 136.7, 137.3, 137.4, 144.1, 167.7. Anal. calcd for $\mathrm{C}_{34} \mathrm{H}_{28} \mathrm{~N}_{2} \mathrm{O}_{2}$ : C 82.23, H 5.68, N 5.64; found $\mathrm{C} 82.30$, H 5.59, N 5.71.

3-(5-甲氧基吲哚基)-3-羰基丙酸乙酯(4b): 白色晶
体, m.p. $122 \sim 124{ }^{\circ} \mathrm{C} ;{ }^{1} \mathrm{H}$ NMR (400 MHz, DMSO- $\left.d_{6}\right) \delta$ : $1.19(\mathrm{t}, J=7.2 \mathrm{~Hz}, 3 \mathrm{H}), 3.78(\mathrm{~s}, 3 \mathrm{H}), 3.96(\mathrm{~s}, 2 \mathrm{H}), 4.08 \sim$ $4.13(\mathrm{q}, J=7.2 \mathrm{~Hz}, 2 \mathrm{H}), 6.85 \sim 6.88(\mathrm{~d}, J=8.8 \mathrm{~Hz}, 1 \mathrm{H})$, 7.80 (d, $J=8.8 \mathrm{~Hz}, 1 \mathrm{H}), 7.66$ (s, 1H), 8.29 (s, 1H), 11.95 $(\mathrm{s}, 1 \mathrm{H}) ;{ }^{13} \mathrm{C} \mathrm{NMR}\left(100 \mathrm{MHz}, \mathrm{DMSO}-d_{6}\right) \delta: 14.1,46.1$, 55.2, 60.4, 102.9, 113.0, 113.0, 115.9, 126.1, 131.5, 135.4, 155.6, 168.2, 187.1. Anal. calcd for $\mathrm{C}_{14} \mathrm{H}_{15} \mathrm{NO}_{4}$ : C 64.36, H 5.79, N 5.36; found C 64.51, H 5.81, N 5.30.

3-(5-溴吲哚基)-3-羰基丙酸乙酯(4c): 白色晶体, m.p. $100 \sim 102{ }^{\circ} \mathrm{C} ;{ }^{1} \mathrm{H}$ NMR (400 MHz, DMSO-d 6 ) $\delta$ : 1.19 (t, $J=7.8 \mathrm{~Hz}, 3 \mathrm{H}), 3.98(\mathrm{~s}, 2 \mathrm{H}), 4.08 \sim 4.13$ (q, $J=$ $7.8 \mathrm{~Hz}, 2 \mathrm{H}), 7.37$ (d, $J=8.4 \mathrm{~Hz}, 1 \mathrm{H}), 7.47$ (d, $J=8.4 \mathrm{~Hz}$, $1 \mathrm{H}), 8.28(\mathrm{~s}, 1 \mathrm{H}), 8.41(\mathrm{~s}, 1 \mathrm{H}), 12.24(\mathrm{~s}, 1 \mathrm{H}) ;{ }^{13} \mathrm{C} \mathrm{NMR}$ (100 MHz, DMSO- $\left.d_{6}\right) \delta: 14.07,46.14,60.48,114.42$, $114.85,115.50,123.33,125.74,127.05,135.47,136.46$, 168.02, 187.39. Anal. calcd for $\mathrm{C}_{13} \mathrm{H}_{12} \mathrm{BrNO}_{3}: \mathrm{C} 50.34, \mathrm{H}$ 3.90, N 4.52; found C 50.22, H 3.81, N 4.62.

3-(1-苠基吲哚基)-3-羰基丙酸乙酯(4e): 白色晶体, m.p. $133 \sim 135{ }^{\circ} \mathrm{C} ;{ }^{1} \mathrm{H}$ NMR (400 MHz, DMSO- $\left.d_{6}\right) \delta$ : $1.18(\mathrm{t}, J=7.8 \mathrm{~Hz}, 3 \mathrm{H}), 3.98(\mathrm{~s}, 2 \mathrm{H}), 4.09 \sim 4.14(\mathrm{q}, J=$ $7.2 \mathrm{~Hz}, 2 \mathrm{H}), 5.52(\mathrm{~s}, 2 \mathrm{H}), 7.23 \sim 7.36(\mathrm{~m}, 7 \mathrm{H}), 7.56(\mathrm{~d}, J=$ $8.8 \mathrm{~Hz}, 1 \mathrm{H}), 8.18$ (d, $J=8.4 \mathrm{~Hz}, 1 \mathrm{H}), 8.56$ (s, 1H), 11.64 $(\mathrm{s}, 1 \mathrm{H}) ;{ }^{13} \mathrm{C}$ NMR $\left(100 \mathrm{MHz}, \mathrm{DMSO}-d_{6}\right) \delta: 14.1,46.3$, $49.9,60.5,111.3,115.4,115.5,121.5,122.5,123.3,126.0$, 127.3 (2C), 127.8, 128.7, 136.7, 136.9, 138.3, 168.1, 186.9. Anal. calcd for $\mathrm{C}_{20} \mathrm{H}_{19} \mathrm{NO}_{3}$ : C 74.75, $\mathrm{H}$ 5.96, N 4.36; found $\mathrm{C} 74.85, \mathrm{H} 6.01, \mathrm{~N} 4.27$.

3-吲哚基-3-羰基丙酸甲酯(4f): 白色晶体, m.p. 133 $135{ }^{\circ} \mathrm{C} ;{ }^{1} \mathrm{H}$ NMR (600 MHz, $\left.\mathrm{CDCl}_{3}\right) \delta: 3.73$ (s, 3 H), $3.89(\mathrm{~s}, 2 \mathrm{H}), 7.26 \sim 7.31(\mathrm{~m}, 2 \mathrm{H}), 7.42(\mathrm{~d}, J=7.4 \mathrm{~Hz}$, 1H), 7.87 (s, 1H), 8.36 (d, $J=7.4 \mathrm{~Hz}, 1 \mathrm{H}), 9.37$ (s, 1H); ${ }^{13} \mathrm{C}$ NMR $\left(150 \mathrm{MHz}, \mathrm{CDCl}_{3}\right) \delta: 46.9,52.5,111.8,117.2$, $122.2,123.0,124.1,125.4,133.0,136.5,168.7,187.1$. Anal. calcd for $\mathrm{C}_{12} \mathrm{H}_{11} \mathrm{NO}_{3}$ : C 66.35, H 5.10, N 6.45; found C 66.52, H 5.01, N 6.39.

3-(1-甲基吲哚基)-3-羰基丙酸甲酯(4g)：白色晶体， m.p. 97 98 ${ }^{\circ} \mathrm{C} ;{ }^{1} \mathrm{H}$ NMR $\left(600 \mathrm{MHz}, \mathrm{CDCl}_{3}\right) \delta$ : $3.73(\mathrm{~s}$, $3 \mathrm{H}), 3.83(\mathrm{~s}, 3 \mathrm{H}), 3.85(\mathrm{~s}, 2 \mathrm{H}), 7.31 \sim 7.33(\mathrm{~m}, 3 \mathrm{H}), 7.74$ $(\mathrm{s}, 1 \mathrm{H}), 8.35 \sim 8.37(\mathrm{~m}, 1 \mathrm{H}) ;{ }^{13} \mathrm{C} \mathrm{NMR}\left(150 \mathrm{MHz}, \mathrm{CDCl}_{3}\right)$ $\delta$ : 33.7, 47.2, 52.5, 109.8, 115.9, 122.6, 123.0, 123.8, $126.3,136.5,137.6,168.6,186.0$. Anal. calcd for $\mathrm{C}_{13} \mathrm{H}_{13} \mathrm{NO}_{3}$ : C 67.52, H 5.67, N 6.06; found $\mathrm{C} 67.38, \mathrm{H}$ $5.77, \mathrm{~N} 6.01$.

3-(1-苠基吲哚基)-3-羰基丙酸甲酯(4h)：无色粘稠 液体. ${ }^{1} \mathrm{H} \mathrm{NMR}\left(600 \mathrm{MHz}, \mathrm{CDCl}_{3}\right) \delta: 3.54(\mathrm{~s}, 2 \mathrm{H}), 3.80(\mathrm{~s}$, 
$3 \mathrm{H}), 5.36(\mathrm{~s}, 2 \mathrm{H}), 7.15 \sim 7.18(\mathrm{~m}, 4 \mathrm{H}) .7 .31 \sim 7.36(\mathrm{~m}$, $5 \mathrm{H}), 7.89$ (d, $J=7.8 \mathrm{~Hz}, 1 \mathrm{H}) ;{ }^{13} \mathrm{C} \mathrm{NMR}\left(150 \mathrm{MHz}, \mathrm{CDCl}_{3}\right)$ $\delta$ : 50.3, 51.0, 65.9, 110.2, 114.3, 120.4, 121.0, 122.9, 126.6, 126.9 (2C), 128.2, 129.0, 129.9 (2C), 136.6, 136.7, 153.5, 166.7. Anal. calcd for $\mathrm{C}_{19} \mathrm{H}_{17} \mathrm{NO}_{3}$ : C 74.25, H 5.58, N 4.56; found C 74.38, H 5.67, N 4.52.

\section{References}

[1] Nicolaou, K. C.; Snyder, S. A. Classics in Total Synthesis II, Wiley-VCH, Weinheim, 2003.

[2] Zhang, G. Z.; Huang, X. G.; Li, G. T. J. Am. Chem. Soc. 2008, $130(6), 1814$.

[3] Ishikawa, H.; Colby, D. A.; Boger, D. L. J. Am. Chem. Soc. 2008, $130(2), 420$.

[4] Jiao, L.; Herdtweck, E.; Bach, T. J. Am. Chem. Soc. 2012, 134(35), 14563.

[5] Phipps, R. J.; Grimster, N. P.; Gaunt, M. J. J. Am. Chem. Soc. 2008, $130(26), 8172$.

[6] Ma, S. M.; Yu, S. C. Org. Lett. 2005, 7(22), 5063.

[7] Zhang, L.; Zhu, Y. X.; Yin, G. W. J. Org. Chem. 2012, 77(21), 9510.

[8] Gao, J.; Wu, H.; Xiang, B. J. Am. Chem. Soc. 2013, $135(8), 2983$.

[9] Łyżwa, D.; Dudziński, K.; Kwiatkowski, P. Org. Lett. 2012, 14(6), 1540 .

[10] Blay, G.; Cano, J.; Cardona, L. J. Org. Chem. 2012, 77(23), 10545.

[11] Garcia-Rubia, A.; Arrayas, R. G.; Garretero, J. C. Angew. Chem., Int. Ed. 2009, 48(35), 6511.

[12] Grimster, N. P.; Gauntlett, C.; Godfrey, C. R. A. Angew. Chem., Int.
Ed. 2005, 44(20), 3125.

[13] Ottoni, O.; Neder, A.; Dias, A. K. B. Org. Lett. 2001, 3(7), 1005.

[14] Pan, L.; Liu, Q. Synlett 2011, 1073.

[15] Junjappa, H.; Ila, H.; Asokan, C. V. Tetrahedron 1990, 46(15), 5423.

[16] Dieter, R. K. Tetrahedron 1986, 42(16), 3029.

[17] Zhang, L.; Liang, F. S.; Cheng, X. J. Org. Chem. 2009, 74(2), 899.

[18] Bi, X. H.; Dong, D. W.; Liu Q. J. Am. Chem. Soc. 2005, 127(13), 4578.

[19] Wang, M.; Sun, S. G.; Liang, D. Q. Eur. J. Org. Chem. 2011, 13, 2466.

[20] Liang, D. Q.; Wang, M.; Bekturhun, B. Adv. Synth. Catal. 2010, $352(10), 1593$.

[21] Yu, H. F.; Jin, W. W.; Sun, C. L. Angew. Chem., Int. Ed. 2010, 49, 5792.

[22] Jin, W. W.; Du, W. M.; Yang, Q. Org Lett. 2011, 13(16), 4272.

[23] Dong, Y.; Wang, M.; Liu, J. Chem. Commun. 2011, 47, 7380.

[24] Yu, H. F. Synth. Commun. 2013, 43, 1280.

[25] Yu, H. F.; Liao, P. Q. Chemistry 2013, (5), 435 (in Chinese). (于海丰, 廖沛球, 化学通报, 2013, (5), 435.)

[26] Yu, H. F. Chin. J. Chem. 2012, 23, 367.

[27] Yu, H. F.; Liao, P. Q. Chem. J. Chin. Univ. 2012, 33, 1969 (in Chinese).

(于海丰, 廖沛球, 高等学校化学学报, 2012, 33 1969.)

[28] Yu, H. F.; Wang, D. L.; Zhao, H.; Hou, D. Y. Chin. J. Org. Chem. 2011, $31(11), 949$ (in Chinese).

(于海丰, 王东来, 赵辉, 侯冬岩, 有机化学, 2011, 31(11), 949.)

[29] Yu, H. F.; Yu, Z. K. Angew. Chem., Int. Ed. 2009, 48, 2929.

[30] Yu, H. F.; Li, T. C.; Liao, P. Q. Synthesis 2012, 3743.

[31] Hinman, R. L.; Shull, E. R. J. Org. Chem. 1961, 26, 2339.

(Li, L.; Fan, Y.) 